\title{
Role of ${ }^{18}$ F-FDG PET/CT in Management of High-Grade Salivary Gland Malignancies
}

\author{
Han-Sin Jeong*1, Man Ki Chung*1, Young-Ik Son ${ }^{1}$, Joon Young $\mathrm{Choi}^{2}$, Hyung Jin $\mathrm{Kim}^{3}$, Young Hyeh $\mathrm{Ko}^{4}$, and \\ Chung-Hwan Baek ${ }^{1}$ \\ ${ }^{I}$ Department of Otorhinolaryngology-Head and Neck Surgery, Samsung Medical Center, Sungkyunkwan University School of Medicine, \\ Seoul, Korea; ${ }^{2}$ Department of Nuclear Medicine, Samsung Medical Center, Sungkyunkwan University School of Medicine, Seoul, Korea; \\ ${ }^{3}$ Department of Radiology, Samsung Medical Center, Sungkyunkwan University School of Medicine, Seoul, Korea; and \\ ${ }^{4}$ Department of Pathology, Samsung Medical Center, Sungkyunkwan University School of Medicine, Seoul, Korea
}

The role of ${ }^{18} \mathrm{~F}-\mathrm{FDG} \mathrm{PET} / \mathrm{CT}$ for planning the treatment of highgrade salivary gland malignancies was investigated and was compared with that with using contrast-enhanced CT. Methods: The subjects chosen for the study had high-grade cancer of the salivary gland, as confirmed by surgical pathology. The diagnostic values from $37 \mathrm{CT}$ and PET/CT scans of 33 subjects were compared. The ability to predict the extent of the disease was compared by performing a subsite-based analysis for the primary lesions and a level-by-level analysis for the neck node levels as well as for the final TNM staging. The surgical pathology $(67.6 \%)$ and clinical follow-up examinations (32.4\%) were used as the reference standards. Furthermore, the changes made in each subject's care, based on a PET/CT examination, were compared with the treatment received without using the PET/CT data. Results: Using a primary subsite-based analysis, the diagnostic accuracy for predicting the pathologic tumor extent was significantly higher for PET/CT (91.0\%) compared with that using CT alone $(70.1 \%, P<0.001)$. For the neck nodes on a level-bylevel analysis, the metastasis could be predicted more accurately on the basis of a PET/CT examination (97.6\%) than with using only CT (86.0\%, $P=0.01)$. PET/CT was also far superior to $\mathrm{CT}$ in terms of the TNM staging $(83.7 \%$ vs. $62.1 \%, P=$ 0.03 ). For $43.2 \%$ of the subjects, changes in the clinical decision making were made as a result of the PET/CT scan data over what was previously determined by using the CT scans alone. Conclusion: PET/CT provides more accurate diagnostic information for the evaluation of high-grade salivary cancer than does CT and it has a major impact on making treatment decisions for patients with a high-grade salivary malignancy.

Key Words: ${ }^{18} \mathrm{~F}-\mathrm{FDG}$; PET/CT; CT scans; salivary gland cancers; treatment

J Nucl Med 2007; 48:1237-1244

DOI: 10.2967/jnumed.107.041350

\footnotetext{
Received Mar. 1, 2007; revision accepted Apr. 20, 2007.

For correspondence or reprints contact: Chung-Hwan Baek, MD, PhD, Department of Otorhinolaryngology-Head and Neck Surgery, Samsung Medical Center, Sungkyunkwan University School of Medicine, 50 Ilwondong, Gangnam-gu, 135-710, Seoul, Korea.

E-mail: chbaek@smc.samsung.co.kr

${ }^{*}$ Contributed equally to this work.

COPYRIGHT @ 2007 by the Society of Nuclear Medicine, Inc.
}

O comprise $<0.5 \%$ of all malignancies and about $5 \%$ of the cancers of the head and neck (1). Although many factors affect the prognosis of patients with salivary gland cancer, the 2 most significant factors are known to be the histologic tumor grade and the clinical stage at presentation $(2,3)$. To achieve an acceptable treatment outcome for high-grade cancer of the salivary gland, it is essential to accurately evaluate the extent of the disease before deciding on the treatment regimen. Thus, various factors such as local invasion of the primary tumors, regional lymph node metastasis, and distant spread of disease need to be assessed.

The conventional pretreatment work-up for salivary gland cancer depends primarily on the information gleaned from contrast-enhanced CT scans and MRI scans. Some studies have reported that CT and MRI provide a similar diagnostic accuracy with respect to the presurgical planning (4-6), although other studies have reported that MRI is superior to CT $(7,8)$. CT has high sensitivity $(>90 \%)$, but it has relatively low specificity (around 60\%), with respect to its ability to distinguish between benign and malignant tumors of the salivary gland (4). However, on the basis of the information from various signal intensities, MRI is superior to CT in terms of sensitivity (nearly 100\%) and specificity $(80 \%)$, in distinguishing benign from malignant tumors (9).

${ }^{18} \mathrm{~F}-\mathrm{FDG}$ PET has been reported to be more accurate than CT or MRI for the detection of malignant tissues, whereas the glucose uptake levels show a good correlation with the histologic grading for head and neck cancer (10). However, for salivary gland cancer, ${ }^{18} \mathrm{~F}$-FDG PET shows low accuracy for distinguishing between benign and malignant tumors as benign tumors such as pleomorphic adenoma and Warthin's tumors also have high glucose uptake values (11-14). According to these studies, the role of ${ }^{18} \mathrm{~F}-\mathrm{FDG}$ PET for the diagnosis of salivary gland cancer is disappointing. Integrated ${ }^{18}$ F-FDG PET/CT is also poor at distinguishing between benign and malignant tumors of the salivary gland (15). By contrast, another study recently concluded that 
${ }^{18}$ F-FDG PET does have a significant impact on the management of patients with salivary gland cancers for both the initial staging and the restaging (16). Moreover, ${ }^{18} \mathrm{~F}-\mathrm{FDG}$ PET could detect cervical lymph node metastases more accurately and it could detect distant metastases and second primary tumors that could not be detected with using a conventional imaging approach (16).

However, little information is available on the diagnostic role of PET/CT for high-grade salivary cancers. For highgrade salivary gland cancers, accurate information relating to the tumor's extent and its spread is very important for planning treatment (17). Accordingly, in this study, the role of integrated ${ }^{18} \mathrm{~F}-\mathrm{FDG}$ PET/CT in the management of salivary gland cancer was investigated, with a focus on high-grade tumors, and this was compared with that using contrast-enhanced CT.

\section{MATERIALS AND METHODS}

Approval of the Institutional Review Board of Samsung Medical Center was obtained for this study. Patient informed consent was not required for this retrospective analysis, but written informed consent was obtained from all patients for performing the integrated ${ }^{18} \mathrm{~F}-\mathrm{FDG}$ PET/CT and contrast-enhanced CT scans.

\section{Subjects}

Thirty-five subjects with high-grade salivary carcinoma were retrieved from the Salivary Gland Malignancy Data Registry at Samsung Medical Center (2002-2005). All pathologic diagnoses were reconfirmed by intradepartment consultations, and the equivocal subjects were discussed with other pathologists to confirm the final pathologic diagnoses. Two subjects were excluded from the pathologic review because the surgical pathology did not meet the criteria of high-grade malignancy of the salivary gland (they were intermediate-grade mucoepidermoid carcinomas). For 14 subjects, high-grade malignancies were prediagnosed on the basis of cytologic analyses, and surgical pathology reconfirmed the high-grade cancer status after surgery. For the remaining 23 subjects, the pathologic diagnoses of high-grade malignancy were confirmed after surgery. Finally, 33 subjects with high-grade salivary carcinoma were included in the analyses, and a total of 37 PET/CT scans with contrast-enhanced CT were obtained (Table 1). Twenty-three imaging studies were performed to evaluate recurrence during the follow-up period, whereas 14 imaging studies were aimed at arriving at the initial diagnosis. Twentythree PET/CT studies for the evaluation of recurrence were performed on 19 patients. All patients underwent surgical treatment as an initial therapy, and the mean duration between the initial treatment and the PET/CT study ranged from 1 to $180 \mathrm{mo}$ (median, $16 \mathrm{mo}$ ). In 6 patients, malignancy was not suggested before the surgery; therefore, PET/CT was performed 1 mo after surgery. Among a total of 37 PET/CT studies, the reference standards were surgical specimens in 25 studies, including 19 patients who underwent neck dissection with or without surgical treatment of the salivary glands. The rest of the studies relied on the clinical follow-up along with the physical examination and other imaging modalities.

The pathologic diagnoses consisted of 5 salivary duct carcinomas, 6 high-grade mucoepidermoid carcinomas, 10 solid-type adenoid cystic carcinomas, 3 squamous cell carcinomas, 3 ade-
TABLE 1

Subject Characteristics $(n=33)$

\begin{tabular}{|c|c|}
\hline Characteristic & No. (\%) \\
\hline $\mathrm{M} / \mathrm{F}$ & $22 / 11(66.7 / 33.3)$ \\
\hline Age (y) & $54(31-83)$ \\
\hline \multicolumn{2}{|l|}{ Site } \\
\hline Parotid gland & $22(66.7)$ \\
\hline Submandibular gland & $7(21.2)$ \\
\hline Minor salivary gland & $4(12.1)$ \\
\hline \multicolumn{2}{|l|}{ Pathology } \\
\hline Salivary duct carcinoma & $5(15.2)$ \\
\hline Mucoepidermoid carcinoma, high grade & $6(18.2)$ \\
\hline Squamous cell carcinoma & $3(9.1)$ \\
\hline Adenoid cystic carcinoma, solid type & $10(30.3)$ \\
\hline $\begin{array}{l}\text { Carcinoma ex pleomorphic } \\
\text { adenoma }\end{array}$ & $6(18.2)$ \\
\hline Adenocarcinoma & $3(9.1)$ \\
\hline \multicolumn{2}{|l|}{ TNM stage } \\
\hline $\mathrm{T} 1-\mathrm{T} 2$ & $19(57.6)$ \\
\hline T3-T4 & $14(42.4)$ \\
\hline NO-N1 & $27(81.8)$ \\
\hline N2-N3 & $6(18.2)$ \\
\hline Mo & $26(78.8)$ \\
\hline M1 & $7(21.2)$ \\
\hline \multicolumn{2}{|l|}{ Treatment modality } \\
\hline $\mathrm{Op}+\mathrm{RT}$ & $26(78.8)$ \\
\hline Chemotherapy & $7(21.2)$ \\
\hline \multicolumn{2}{|l|}{ Treatment outcome } \\
\hline NED & $15(45.5)$ \\
\hline AWD & $13(39.4)$ \\
\hline DOD & $5(15.2)$ \\
\hline Follow-up (mo) & $24.3(12-47)$ \\
\hline \multicolumn{2}{|l|}{ PET/CT and CT work-up $(n=37)$} \\
\hline Initial evaluation & $14(37.8)$ \\
\hline Follow-up & $23(62.2)$ \\
\hline
\end{tabular}

$\mathrm{Op}=$ operation; $\mathrm{RT}=$ radiation therapy; NED = no evidence of disease; AWD = alive with disease; DOD = died of disease.

nocarcinomas, and 6 carcinoma ex pleomorphic adenoma. The patients' ages ranged from 31 to $83 \mathrm{y}$ (mean, $54.48 \mathrm{y}$ ); 22 patients were male and 11 were female. The majority of the primary salivary cancers arose from the parotid gland (66.7\%), whereas some were from the submandibular gland $(21.2 \%)$ and the minor salivary glands $(12.1 \%)$, including those in the lip and soft palate and 2 sublingual glands. The initial $\mathrm{T}$ classifications of the subjects were T1-T2 for 19 patients and T3-T4 for 14 patients. At the initial diagnosis, N0-N1 was the most frequent nodal status of the subjects (81.8\%); however, distant metastasis (lung, iliac bone, and paraaortic lymph nodes) was detected in 7 patients (21.2\%). During the follow-up, another patient was detected to have distant metastasis on PET/CT.

All patients without distant metastasis were subjected to curative surgical resection with postoperative radiation therapy, whereas 7 patients with distant metastasis underwent palliative chemotherapy. As for the treatment outcome, 18 patients $(54.5 \%)$ had distant metastasis (lung, liver, bone, abdominal lymph nodes), including 8 subjects that were diagnosed by PET/CT. Unfortunately, PET/CT was not performed on the remaining 10 patients when distant metastasis was detected with the help of conventional studies, so comparative analysis was not available. 
The average follow-up period of the study was 24.3 mo (range, 12-47 mo).

\section{Contrast-Enhanced CT Scans}

CT scans (LightSpeed Ultra or Ultra 16; GE Healthcare) of the head and neck, focusing on the sites of the salivary gland, were performed using the following parameters: $160 \mathrm{~mA} \cdot \mathrm{s}, 120 \mathrm{keV}$, a section width of $3.75 \mathrm{~mm}$, and a table feed of $8.75 \mathrm{~mm}$ per rotation. For contrast enhancement, $90 \mathrm{~mL}$ of an iodinated contrast agent (Iopromide, Ultravist 300; Schering) were injected intravenously at $3 \mathrm{~mL} / \mathrm{s}$ using an automated injector. The scan delay time was $30 \mathrm{~s}$.

\section{Integrated ${ }^{18}$ F-FDG PET/CT Scans}

All patients fasted for at least $6 \mathrm{~h}$ before PET/CT, which were performed using a Discovery LS PET/CT scanner (GE Healthcare). Whole-body CT was performed by a continuous spiral technique and using 8-slice helical CT with a gantry rotation speed of $0.8 \mathrm{~s}$. The CT scan data were collected using the following parameters: $80 \mathrm{~mA} \cdot \mathrm{s}, 140 \mathrm{keV}$, a section width of $5 \mathrm{~mm}$, and a table feed of $5 \mathrm{~mm}$ per rotation. No intravenous or oral contrast agents were used. After the CT scans and after injecting $370 \mathrm{MBq}$ ${ }^{18} \mathrm{~F}$-FDG intravenously, an emission scan was performed from the thigh to the head for $5 \mathrm{~min}$ per frame, for a total of $45 \mathrm{~min}$. The duration of the uptake phase was $45 \mathrm{~min}$. The attenuationcorrected ${ }^{18} \mathrm{~F}$-FDG PET images were reconstructed using the CT data with an ordered-subset expectation maximization algorithm (28 subsets, 2 iterations). The images were displayed in a $128 \times$ 128 matrix (pixel size $=4.29 \times 4.29 \mathrm{~mm}$, with a slice thickness of $4.25 \mathrm{~mm}$ ). The separate CT and PET scan data were coregistered accurately using commercial software (eNTEGRA; Elgems). The standardized uptake values (SUVs) were acquired using the attenuation-corrected images, the amount of injected ${ }^{18} \mathrm{~F}-\mathrm{FDG}$, the body weight of each patient, and the cross-calibration factors between ${ }^{18} \mathrm{~F}$-FDG PET and the dose calibrator.

\section{Image Analysis}

For the subsite-based analysis, we divided the structures around the salivary gland into subsites according to the American Joint Committee on Cancer Staging (AJCC) Manual criteria (18): the primary tumor size, the extraparenchymal extension and the involvement of skin, the mandible or maxilla bone, the ear canal, the skull base, and the pterygoid plate. If each diagnostic modality predicted the malignant tumors in the affected gland and the pathologic tumor size within an error of $1 \mathrm{~cm}$ for the diameter, then this was considered as a true-positive for tumor size. The regional cervical nodes were also subdivided into the cervical lymph node levels (levels I-VI, the periparotid and infraparotid lymph node groups).

For the CT scans, a radiologist who specializes in interpreting head and neck sections determined the extent of the salivary gland lesion and the significance of the cervical lymph nodes, without having any knowledge of the clinical findings. First, the interpretation of the CT scans was focused on the primary lesions of the salivary gland. Salivary lesions with an abnormal enhancement pattern were evaluated to assess the tumor extent, according to the subsites. The regional lymph nodes of the head and neck region were also evaluated according to the accepted criteria (19).

For PET/CT, 1 nuclear medicine physician, who was experienced in interpreting head and neck imaging for $>5 \mathrm{y}$, reviewed the fused PET/CT images without having any knowledge of the clinical findings. The nuclear medicine physician first reviewed the images to determine any abnormal uptake of ${ }^{18} \mathrm{~F}-\mathrm{FDG}$ in the salivary gland and neck nodes by using the maximal uptake values with intensity that was higher than that of the surrounding tissues. An abnormal location and asymmetry of the ${ }^{18}$ F-FDG uptake was also considered as an important diagnostic criterion for PET/CT. Their interpretation was then revised on the basis of the anatomic information provided by the combined PET/CT images. For example, a nonnodal focal uptake in a blood vessel or muscle that mimicked a lymph node was considered benign. A lymph node with low peripheral attenuation suggesting a fatty hilum on the CT image was considered benign even if it had a high ${ }^{18} \mathrm{~F}-\mathrm{FDG}$ uptake. On the contrary, a lymph node containing a lucent portion on the CT image and low ${ }^{18} \mathrm{~F}-\mathrm{FDG}$ uptake on the PET image that was suggestive of necrosis was considered malignant even if it had a low ${ }^{18}$ F-FDG uptake.

Similar to the interpretation of the CT scans, a nuclear physician determined the status of each subsite from the information of the "stand-alone" PET/CT scans. The interpretation of each subsite was classified as malignant-positive, malignant-negative, or equivocal. During PET/CT, there were 2 equivocal subsites in the evaluation of primary tumors and 9 equivocal sites in the evaluation of distant metastasis. There was no equivocal lesion in the evaluation of the nodes at the neck level. During CT, 4 equivocal lesions were measured when evaluating the distant metastasis. The equivocal subjects were reclassified into malignant-positive or malignant-negative after separately reviewing the images by the same radiologists or the nuclear medicine physician.

\section{Decision Making for Treatment}

The Head and Neck Cancer Tumor Board decided the treatment plan for the salivary lesions after a review of the results of the clinical examinations and the CT scans. The team then reviewed the PET/CT images, and any changes in the decision making for treatments were recorded. The final treatment plans were determined after a discussion with each patient, and the available treatment options were outlined.

\section{Statistical Analysis}

The reference standards for describing the status of each subsite and the initial tumor stage were derived from the histopathologic results from the biopsies and the surgical excisions in 25 subjects. The clinical and radiologic follow-up for neck nodes and distant sites were also used as a reference in 8 patients.

We determined the sensitivity, specificity, positive predictive value (PPV), negative predictive value (NPV), and diagnostic accuracy of CT and PET/CT for predicting the extent of disease using the subsite-based analysis that was described. Statistical differences between the imaging modalities were analyzed by using the McNemar test, and the 95\% confidence levels were determined using Wilson's method. Two-tailed $P$ values $<0.05$ were considered to be statistically significant. The clinical decisions for treatment using CT alone and then adding PET/CT were recorded to register the impact of each diagnostic modality on patient care. The additional benefits provided by PET/CT were noted.

\section{RESULTS}

\section{Diagnostic Values of Subsite-Based Analysis for High-Grade Salivary Cancer}

The maximum SUV of the primary lesions ranged from 1.5 to 23.7, with a mean SUV \pm SD of $8.21 \pm 3.62$ (Table 2). A minimum SUV of 1.5 was noted for the adenoid cystic 
TABLE 2

Maximum SUV of Primary Sites According to Pathologic Type of Cancer

\begin{tabular}{lc}
\hline \multicolumn{1}{c}{ Pathologic type of cancer } & Range of maximum SUV \\
\hline Salivary duct carcinoma & $8.6-11.3$ \\
Mucoepidermoid carcinoma & $5.3-23.7$ \\
Adenoid cystic carcinoma & $1.5-6.2$ \\
Squamous cell carcinoma & $5.5-16.8$ \\
Adenocarcinoma & $7.2-11.4$ \\
Carcinoma ex pleomorphic adenoma & $7.6-14.1$ \\
\hline
\end{tabular}

carcinoma at the submandibluar gland, and a maximum SUV of 23.7 was detected for the mucoepidermoid carcinoma at the submandibular gland.

Within the primary site, there was a total of 67 subsites as revealed by 37 PET/CT and CT scans that were then evaluated (Table 3). Of these, the size of the salivary tumors was analyzed by PET/CT and CT to predict the pathologic tumor size, and this was achieved to within an error of $1 \mathrm{~cm}$ for the diameter in 21 subjects. When comparing PET/CT and CT for predicting the pathologic primary tumors, CT had a sensitivity of $64.2 \%$, a specificity of $80.0 \%$, a PPV of $84.3 \%$, a NPV of $57.1 \%$, and a diagnostic accuracy of $70.1 \%$; the corresponding values for the PET/CT scans were $85.7 \%, 100.0 \%, 100.0 \%$, $80.6 \%$, and $91.0 \%$, respectively. Thus, the sensitivity, NPV, and overall diagnostic accuracy were each significantly higher for PET/CT than for CT, whereas the differences in the other parameters were close to being statistically significant.
Figure 1 presented a subject of initial evaluation. There was only a poorly marginated soft-tissue lesion without definite enhancement in the submandibular area, as seen on the CT scan. But PET/CT showed a high SUV uptake at the same area, suggesting malignancy, and this was proven to be true on the surgical pathology. When imaging studies were performed for the evaluation of recurrence, it was more difficult to differentiate inflammation from recurrence. A patient who had surgical treatment and postoperative radiation therapy presented different results on CT and PET/CT during the follow-up (Fig. 2). PET/CT correctly diagnosed the recurrence in this patient.

Eighty-six neck node analyses were done to determine the diagnostic values (Table 4). For predicting cervical lymph node metastasis, CT had a sensitivity of $71.8 \%$, a specificity of $94.4 \%$, a PPV of $88.4 \%$, a NPV of $85.0 \%$, and a diagnostic accuracy of $86.0 \%$; the corresponding values for the PET/CT scans were $100.0 \%, 96.3 \%, 94.1 \%, 100.0 \%$, and $97.6 \%$, respectively. Again, the sensitivity, NPV, and overall diagnostic accuracy were significantly higher for PET/CT than for CT for the evaluation of cervical metastasis (Table 4). On the PET images, the maximum SUV of the neck nodes ranged from 1.4 to 27.2 , with a mean $\mathrm{SUV} \pm \mathrm{SD}$ of $7.07 \pm 5.36$.

In the subject with recurrence at multiple cervical lymph nodes, PET/CT detected the metastatic lymph node at the right highest mediastinum, which made a surgical approach deep to that level necessary (Fig. 3). CT failed to provide definitive information for the same lesion.

TABLE 3

Diagnostic Values of Contrast-Enhanced CT (CT) and PET/CT, Obtained by Primary Subsite-Based Analysis, for Patients with High-Grade Salivary Cancer ( $n=67$ Subsites)

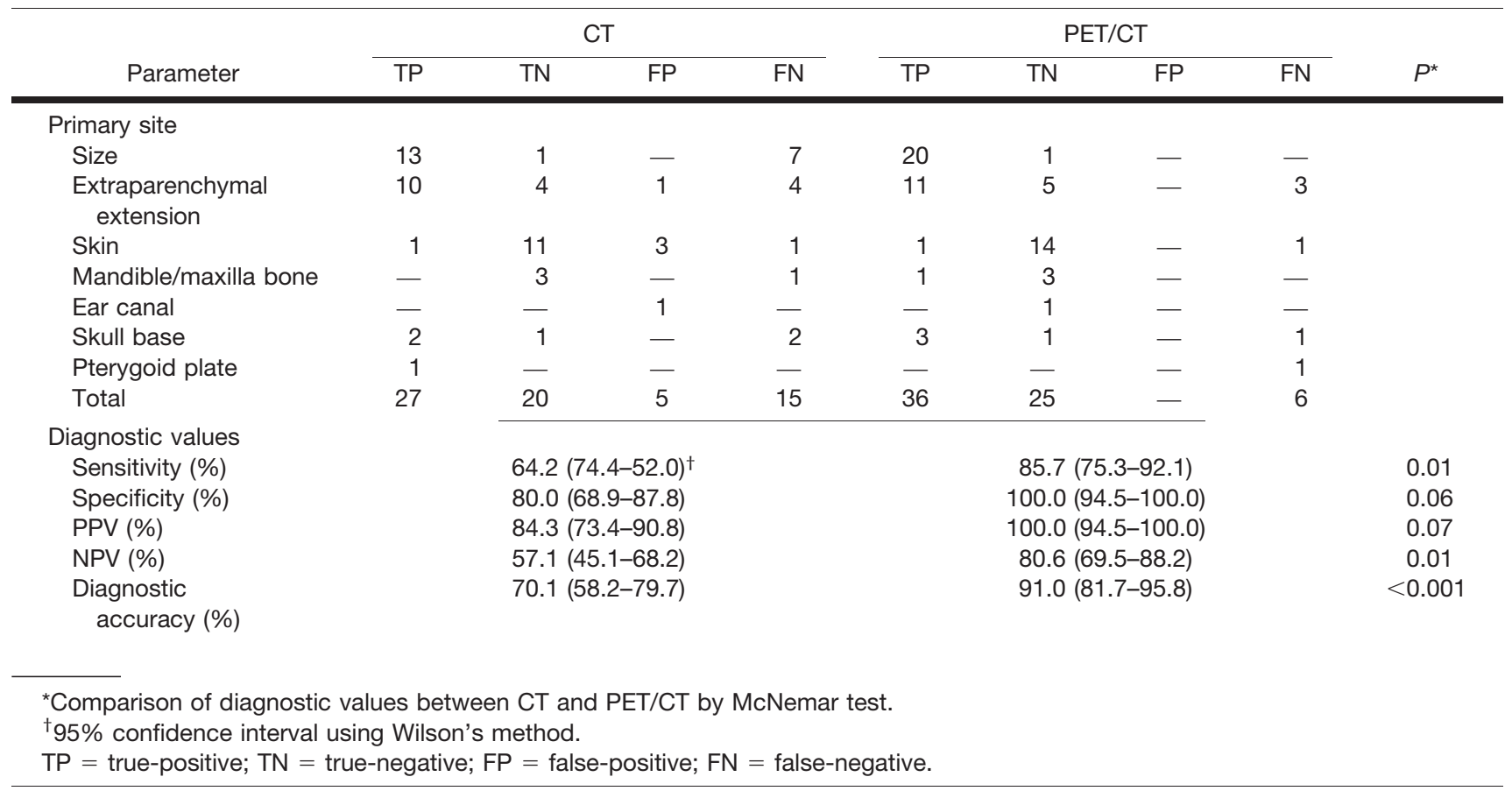



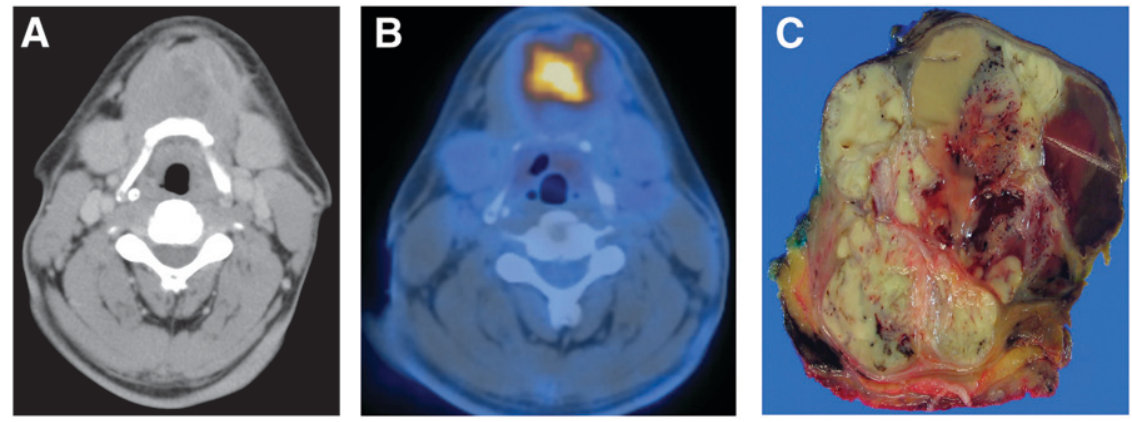

FIGURE 1. A 50-y-old man with adenocarcinoma in sublingual gland. (A) There was no enhancement in mass at floor of mouth; thus, judging from CT scans, mass is classified as benign lesion. (B) Using $\mathrm{PET} / \mathrm{CT}$ scans, lesion showed high glucose metabolism (maximum SUV $=10.6$ ) with irregular margins. Interpretation was that the lesion was malignant tumor. (C) Final surgical pathology showed a $5 \times 4 \mathrm{~cm}$ adenocarcinoma, not otherwise specified.

\section{Diagnostic Accuracy for Determination of TNM Tumor Stages of High-Grade Salivary Cancer}

For predicting the TNM stages, CT had a diagnostic accuracy of $62.1 \%$, as compared with $83.7 \%$ for the PET/ CT scans; thus, PET/CT was far superior to CT $(P=0.03)$ (Table 5).

The $\mathrm{T}$ and $\mathrm{N}$ classifications were determined separately for each diagnostic modality; however, the $\mathrm{M}$ classification was determined from the clinical examination, a routine simple chest X-ray, and the CT scans. In the case of PET/ $\mathrm{CT}$, all $\mathrm{T}, \mathrm{N}$, and $\mathrm{M}$ classifications were determined solely by the results of PET/CT.

\section{Impact on Clinical Treatment Planning}

The clinical decision making with regard to treatment, as based on CT, was modified by adding the information gained from PET/CT in 16 of the 37 subjects we studied $(43.2 \%)$ (Table 6). In 5 patients, the extent of surgery indicated for their primary lesions was modified. In 3 of these patients, CT was not able to detect the primary lesions as malignant tumors. Therefore, the information obtained from PET/CT converted a conservative resection to a comprehensive resection of the primary tumor. For 1 patient, PET/CT detected the recurrence in the surgical bed
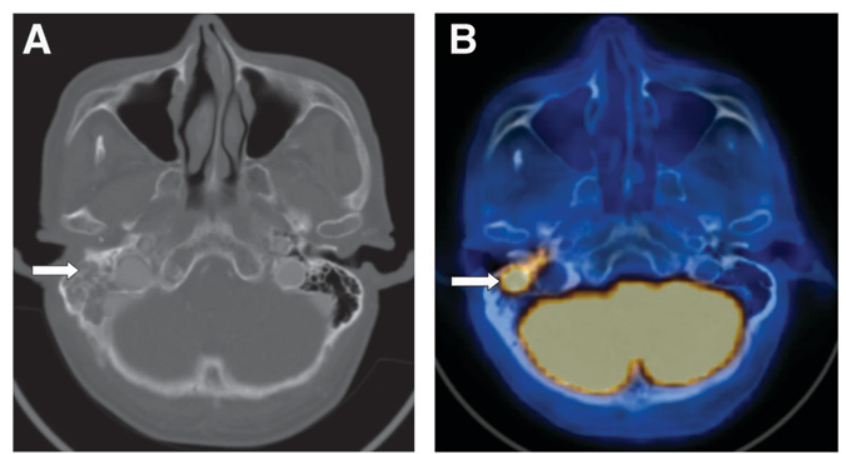

FIGURE 2. A 78-y-old man with salivary duct carcinoma in right parotid gland who underwent CT and PET/CT scans at 24 mo after initial treatment of a wide surgical resection with postoperative radiation therapy. (A) CT scans led to impression of right mastoid inflammation, on basis of finding of an intact bony wall (arrow). (B) In contrast, using PET/CT images, maximum SUV of lesion was 10.8, and tumor invasion into temporal bone was strongly suggested (arrow). Final surgical pathology revealed malignant cells in mastoid bone. after the initial surgery and radiation therapy, and this allowed salvage surgery to be undertaken. The PET/CT information led to decreasing the extent of surgery in 1 patient; thus, a radical parotidectomy was avoided, including that on the periparotid muscles. PET/CT correctly predicted a final T1 tumor, whereas CT had interpreted the T classification of this tumor as $\mathrm{T} 4$.

For the neck nodes, the PET/CT information changed an elective neck dissection regimen in 1 patient to a comprehensive neck dissection. Also for this patient, the peritumor regional lymph node groups (infraparotid and preparotid) were included in the extent of neck dissection, based on the results of the PET/CT scans.

In 8 subjects, PET/CT detected distant metastasis (lung, liver, bone, abdominal lymph nodes); therefore, the treatment was changed from a curative intent to a palliative setting.

\section{DISCUSSION}

The aim of this study was to investigate the role of integrated ${ }^{18} \mathrm{~F}-\mathrm{FDG}$ PET/CT for the management of salivary gland cancer, with special focus on tumors of a high-grade nature. We then compared PET/CT with contrast-enhanced CT. The results demonstrate that the PET/CT image data significantly improved the diagnostic accuracy for evaluating the extent of tumor and the tumor stages compared with CT alone for patients with high-grade salivary cancers. Several previous studies have concluded that ${ }^{18} \mathrm{~F}-\mathrm{FDG}$ PET is superior to conventional imaging modalities for the evaluation of salivary gland tumors or for distinguishing between benign and malignant masses (11-14). A recent report on the use of ${ }^{18} \mathrm{~F}$-FDG PET for the management of patients with salivary gland malignancies also concluded that it could have a significant role in the initial staging and monitoring after treatment (20). However, these authors did not analyze the anatomic localization of the primary tumors on a subsite basis, as was done in our study; they used only the results of PET images.

In the present study, we used the subsite-based analysis rather than the $\mathrm{T}$ or $\mathrm{N}$ classification for more accurate anatomic evaluation. The $\mathrm{T}$ classification is based on the extent of involvement in different, anatomic subsites. We used the concept of subsites of the salivary gland that was taken from the AJCC classification. This made it easier to compare the diagnostic accuracy for the primary lesion 
TABLE 4

Diagnostic Values of Contrast-Enhanced CT (CT) and PET/CT for Cervical Metastasis in Patients with High-Grade Salivary Cancer on a Level-by-Level Analysis ( $n=86$ Node Levels)

\begin{tabular}{|c|c|c|c|c|c|c|c|c|c|}
\hline \multirow[b]{2}{*}{ Parameter } & \multicolumn{4}{|c|}{ CT } & \multicolumn{4}{|c|}{ PET/CT } & \multirow[b]{2}{*}{$P^{*}$} \\
\hline & TP & $\mathrm{TN}$ & $\mathrm{FP}$ & $\mathrm{FN}$ & TP & TN & FP & $\mathrm{FN}$ & \\
\hline \multicolumn{10}{|l|}{ Neck node levels } \\
\hline I & 4 & 14 & 1 & 1 & 5 & 15 & - & - & \\
\hline$\|$ & 5 & 11 & 2 & 3 & 8 & 13 & - & - & \\
\hline III & 4 & 10 & - & 1 & 5 & 10 & - & - & \\
\hline IV & 2 & 7 & - & 2 & 4 & 6 & 1 & - & \\
\hline V & 4 & 6 & - & 1 & 5 & 5 & 1 & - & \\
\hline VI & - & 1 & - & 1 & 1 & 1 & - & - & \\
\hline Others ${ }^{\dagger}$ & 4 & 2 & - & - & 4 & 2 & - & - & \\
\hline Total & 23 & 51 & 3 & 9 & 32 & 52 & 2 & - & \\
\hline \multicolumn{10}{|l|}{ Diagnostic values } \\
\hline Sensitivity (\%) & \multicolumn{4}{|c|}{$71.8(62.5-80.2)^{\ddagger}$} & \multicolumn{4}{|c|}{$100.0(95.7-100.0)$} & 0.003 \\
\hline Specificity (\%) & \multicolumn{4}{|c|}{$94.4(87.3-97.6)$} & \multicolumn{4}{|c|}{96.3 (89.9-98.6) } & NS \\
\hline PPV (\%) & \multicolumn{4}{|c|}{88.4 (79.9-93.5) } & \multicolumn{4}{|c|}{$94.1(86.9-97.4)$} & NS \\
\hline NPV (\%) & \multicolumn{4}{|c|}{$85.0(75.9-91.0)$} & \multicolumn{4}{|c|}{$100.0(95.7-100.0)$} & 0.01 \\
\hline Diagnostic accuracy (\%) & \multicolumn{4}{|c|}{$86.0(77.1-91.7)$} & \multicolumn{4}{|c|}{97.6 (91.8-99.3) } & 0.01 \\
\hline \multicolumn{10}{|c|}{$\begin{array}{l}{ }^{*} \text { Comparison of diagnostic values between CT and PET/CT by McNemar test. } \\
{ }^{+} \text {Periparotid and infraparotid lymph node groups. } \\
{ }^{*} 95 \% \text { confidence interval using Wilson's method. }\end{array}$} \\
\hline
\end{tabular}

using CT. As for the $\mathrm{N}$ classification, we also used a levelby-level analysis for the exact localization of nodal involvement. This subsite analysis of the primary tumors and the cervical nodes provided more useful information for treatment decisions-for example, the extent of surgery or the field of irradiation. For distant sites, we focused primarily on the presence or absence of distant metastasis, which was usually confirmed by subsequent imaging modalities (chest CT, bone MRI), biopsy, or follow-up data. As a result, interpretation of the anatomic extent and the metabolic rate of the tumors as well as a subsite-based analysis for surgical planning were all achieved. It also should be noted that the different histologic grades of salivary malignancies
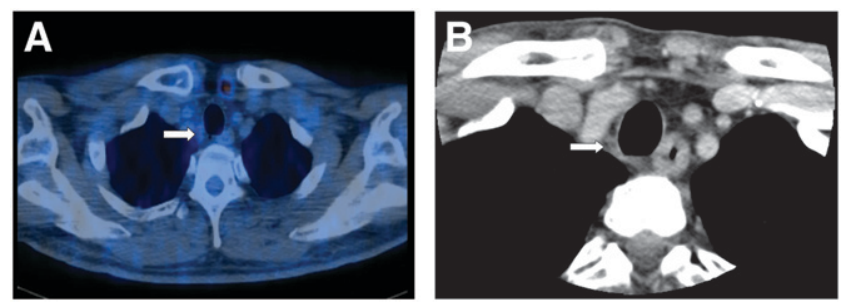

FIGURE 3. CT and PET/CT scans were performed on 52-yold-man with primary squamous cell carcinoma in right parotid gland as initial work-up. (A) PET/CT scans demonstrated right lower anterior lymph node metastasis with a maximum SUV of 2.7 (arrow). (B) In contrast, CT scans failed to detect this regional metastasis, on basis of size of nodes and minimal enhancement pattern (arrow). Malignant cells were found in this node, according to final surgical pathology. Another active ${ }^{18} \mathrm{~F}-$ FDG uptake lesion was evident beside left clavicle; it was correctly diagnosed as malignancy on both CT and PET/CT. were included in a previous report, and this may have affected the interpretation of the PET images (20).

The difference in the results of the previous studies on PET or PET/CT could be partially attributed to subjective characteristics. Thus, if we focus on distinguishing between malignant and benign salivary gland tumors, PET often fails, whereas PET can provide useful information for decision making with respect to the high metastatic potential of salivary gland cancer. In fact, when deciding on a treatment plan, distinguishing between benign tumor and malignant tumor is not too important because low-grade salivary gland cancers appear to show a good prognosis after conservative treatment and this is similar to the subjects with benign salivary tumors. However, for highgrade salivary gland cancers, accurate information on the tumor extent and its spread is more important for planning treatment. In the present study, we focused exclusively on high-grade salivary cancers, which would normally require more aggressive treatment; this is different from low-grade salivary cancers. By this means, we were able to show the impact of a PET/CT examination for patients with highgrade salivary cancers.

There was wide range of maximum SUV values (Table 2), and we assumed that it might be partially due to the variable pathologic characteristics of the salivary cancers, even though they were all categorized as high-grade. Except for the adenoid cystic carcinomas, which are characterized as being relatively slow growing, the other high-grade salivary pathologies showed SUVs of $>5.0$. However, the minimum SUV of 1.5 (adenoid cystic carcinoma) was asymmetrically detected in the submandibular area, which 
TABLE 5

Prediction of Tumor TNM Staging Using ContrastEnhanced CT and PET/CT for Patients with High-Grade Salivary Cancer $(n=37)$

\begin{tabular}{|c|c|c|}
\hline \multicolumn{2}{|c|}{ Diagnostic accuracy (\%) } & \multirow[b]{2}{*}{$P^{*}$} \\
\hline CT & PET/CT & \\
\hline $62.1(48.6-78.1)^{\dagger}$ & $83.7(65.7-90.4)$ & 0.03 \\
\hline \multicolumn{3}{|c|}{ "Test modalities compared using McNemar test. } \\
\hline
\end{tabular}

raised the suggestion of malignancy in the submandibular gland. One case of mucoepidermoid carcinoma had an SUV of 23.7, and the size of the tumor reached $6.5 \mathrm{~cm}$. In this subject, the high SUV was attributable to the huge size of the primary tumor.

The sensitivity and specificity of PET/CT was $85.7 \%-$ $100.0 \%$ and $96.3 \%-100.0 \%$, respectively, with respect to the subsite-based and node level-based analysis. These values were similar to the values of the previous reports on PET usage for primary tumors, but our values were slightly higher for neck nodes, compared with the other reports (20). These differences may be explained on the basis of the subsite-based analysis, which used stricter criteria. For example, prediction of the pathologic size as well as detection of malignant tumors was considered to be true-positive. For evaluating the neck nodes, the alleged CT criteria for distinguishing between benign and malignant cervical lymph nodes-such as the presence of necrotic nodes and nodes with a peripheral fatty hilum - contributed to the improved accuracy using PET/CT for evaluating the cervical nodes combined with the SUV assessment $(19,21)$. This suggests that the CT data are also helpful in the interpretation of PET/CT images for the patients with salivary cancer.

Even though PET/CT showed diagnostic superiority for the management of high-grade salivary cancers, the combined interpretation of contrast-enhanced CT and PET/CT can provide more accurate information about the extent of

TABLE 6

Impact on Patient Care: Contrast-Enhanced CT vs. PET/CT $(n=37)$

\begin{tabular}{lc}
\hline \multicolumn{1}{c}{ Parameter } & $\begin{array}{c}\text { Changes in patient } \\
\text { care by } \\
\text { PET/CT, number }\end{array}$ \\
\hline Extent of surgery for primary lesions & $5(13.5)$ \\
Extent of surgery for neck nodes & $3(8.1)$ \\
Changed curative treatment to & $8(21.6)$ \\
palliative treatment & $16 / 37(43.2)$ \\
Total & \\
\hline Values in parentheses are percentage. & \\
\hline
\end{tabular}

disease. Using combined interpretation of the contrastenhanced CT with PET/CT side by side, the sensitivity, specificity, and diagnostic accuracy for the primary tumors on the subsite-based analysis were $88.1 \%, 100 \%$, and $92.6 \%$, respectively. Similarly, the corresponding values for the neck nodes on the level-by-level analysis were all $100 \%$. Therefore, integrated PET along with contrastenhanced CT may give the best diagnostic results for highgrade salivary malignancies.

With respect to tumor staging, PET/CT was correct in predicting 31 of 37 subjects $(83.7 \%)$, whereas CT was correct in only 23 of 37 subjects $(62.1 \%)$. For the 10 subjects in which CT had incorrectly predicted the tumor staging, the results of PET/CT were correct, as was ultimately assessed by the pathologic results and follow-up data. There were only 2 subjects for which CT was correct and PET/CT was incorrect for the staging of a tumor, whereas for 4 subjects, neither modality provided an accurate estimate.

The PET/CT method has an additional advantage in that it is a whole-body imaging procedure, which may be useful in detecting distant metastases or a second cancer $(22,23)$. The treatment outcome of our study showed the frequent occurrence of distant metastasis in high-grade salivary cancer patients (18/33 subjects, $54.5 \%)$. Moreover, when PET/CT was performed, it was successful in detecting a distant metastasis, as compared with the conventional metastasis detection methods. If PET/CT could fully replace the conventional approach for detecting distant metastasis or a double primary cancer, the high cost of PET/CT would be acceptable and justified.

More importantly, the information from the PET/CT scans had a major impact on the clinical treatment planning in about $40 \%$ of the subjects. For the high-grade salivary gland malignancies, only surgical resection guarantees curative treatment, and the role of other treatments, such as chemotherapy or radiotherapy, is confined to an adjuvant or palliative intent. Therefore, the exact decision on the operability is of utmost importance when evaluating those patients with high-grade salivary gland malignancies. Unlike previous reports, the present study analyzed the impact of PET/CT on the clinical treatment planning and revealed that $21.6 \%$ of the patients were proven to be inoperable because of their distant metastasis; thus, unnecessary surgeries could be avoided for these patients. Even for the operable patients, the increased anatomic accuracy of PET/CT changed the extent of surgery on the primary lesions and neck nodes (13.5\% and $8.1 \%$, respectively); such findings helped enhance the possibility of cure for patients with high-grade salivary gland malignancies.

\section{CONCLUSION}

Our results indicate that ${ }^{18} \mathrm{~F}-\mathrm{FDG}$ PET/CT provides superior diagnostic accuracy for evaluating the extent of tumors and for tumor staging, as compared with CT scans, for the patients with high-grade salivary cancer. Moreover, 
the use of PET/CT has a major positive impact on the clinical decision-making process for those patients who have these cancers.

\section{REFERENCES}

1. Speight PM, Barrett AW. Salivary gland tumours. Oral Dis. 2002;8:229-240.

2. Kokemueller H, Swennen G, Brueggemann N, Brachvogel P, Eckardt A, Hausamen JE. Epithelial malignancies of the salivary glands: clinical experience of a single institution-a review. Int J Oral Maxillofac Surg. 2004; $33: 423-432$

3. Lima RA, Tavares MR, Dias FL, et al. Clinical prognostic factors in malignant parotid gland tumors. Otolaryngol Head Neck Surg. 2005;133:702-708.

4. Kim KH, Sung MW, Yun JB, et al. The significance of CT scan or MRI in the evaluation of salivary gland tumors. Auris Nasus Larynx. 1998;25: 397-402.

5. Arbab AS, Koizumi K, Toyama K, et al. Various imaging modalities for the detection of salivary gland lesions: the advantages of ${ }^{201} \mathrm{Tl}$ SPET. Nucl Med Commun. 2000;21:277-284.

6. Koyuncu M, Sesen T, Akan H, et al. Comparison of computed tomography and magnetic resonance imaging in the diagnosis of parotid tumors. Otolaryngol Head Neck Surg. 2003;129:726-732.

7. Tabor EK, Curtin HD. MR of the salivary glands. Radiol Clin North Am. 1989; 27:379-392

8. Traxler M, Hajek P, Solar P, Ulm C. Magnetic resonance in lesions of the parotid gland. Int J Oral Maxillofac Surg. 1991;20:170-174.

9. Yabuuchi H, Fukuya T, Tajima T, Hachitanda Y, Tomita K, Koga M. Salivary gland tumors: diagnostic value of gadolinium-enhanced dynamic MR imaging with histopathologic correlation. Radiology. 2003;226:345-354.

10. Nowak B, Di Martino E, Janicke S, et al. Diagnostic evaluation of malignant head and neck cancer by F-18-FDG PET compared to CT/MRI. Nuklearmedizin. 1999;38:312-318.
11. Keyes JW Jr, Harkness BA, Greven KM, Williams DW 3rd, Watson NE Jr, McGuirt WF. Salivary gland tumors: pretherapy evaluation with PET. Radiology. 1994;192:99-102.

12. McGuirt WF, Keyes JW Jr, Greven KM, Williams DW 3rd, Watson NE Jr, Cappellari JO. Preoperative identification of benign versus malignant parotid masses: a comparative study including positron emission tomography. Laryngoscope. 1995; 105:579-584.

13. Okamura T, Kawabe J, Koyama K, et al. Fluorine-18 fluorodeoxyglucose positron emission tomography imaging of parotid mass lesions. Acta Otolaryngol Suppl. 1998;538:209-213.

14. Uchida Y, Minoshima S, Kawata T, et al. Diagnostic value of FDG PET and salivary gland scintigraphy for parotid tumors. Clin Nucl Med. 2005;30:170-176.

15. Rubello D, Nanni C, Castellucci P, et al. Does ${ }^{18}$ F-FDG PET/CT play a role in the differential diagnosis of parotid masses. Panminerva Med. 2005;47:187-189.

16. Otsuka H, Graham MM, Kogame M, Nishitani H. The impact of FDG-PET in the management of patients with salivary gland malignancy. Ann Nucl Med. 2005; 19:691-694.

17. Lim YC, Lee SY, Kim K, et al. Conservative parotidectomy for the treatment of parotid cancers. Oral Oncol. 2005;41:1021-1027.

18. Greene FL, Page DL, Fleming ID, et al. AJCC Cancer Staging Manual. 6th ed. New York, NY: Springer-Verlag; 2002.

19. Som PM. Detection of metastasis in cervical lymph nodes: CT and MR criteria and differential diagnosis. AJR. 1992;158:961-969.

20. Roh JL, Ryu CH, Choi SH, et al. Clinical utility of ${ }^{18}$ F-FDG PET for patients with salivary gland malignancies. J Nucl Med. 2007;48:240-246.

21. Jeong HS, Baek CH, Son YI, et al. Use of integrated ${ }^{18} \mathrm{~F}-\mathrm{FDG}$ PET/CT to improve the accuracy of initial cervical nodal evaluation in patients with head and neck squamous cell carcinoma. Head Neck. 2007;29:203-210.

22. Choi JY, Lee KS, Kwon OJ, et al. Improved detection of second primary cancer using integrated $\left[{ }^{18} \mathrm{~F}\right]$ fluorodeoxyglucose positron emission tomography and computed tomography for initial tumor staging. J Clin Oncol. 2005;23:7654-7659.

23. Iagaru A, Chawla S, Menendez L, Conti PS. ${ }^{18}$ F-FDG PET and PET/CT for detection of pulmonary metastases from musculoskeletal sarcomas. Nucl Med Commun. 2006;27:795-802. 